\title{
Ueber das Additionstheorem der Abelschen Functionen.
}

\author{
(Von Herrn H. Weber in Heidelberg.)
}

Die in dem vorliegenden Aufsatz mitgetheilten Untersuchungen über das Additionstheorem der allgemeinen Abelschen Functionen beruhen im Wesentlichen auf der Anwendung der Principien von Riemann. Als Begriff der Abelschen Functionen lege ich den aus dem Jacobischen Umkehrproblem hervorgehenden zu Grunde, und aus diesem habe ich auch die Aufgabe der Addition entnommen. Ich bin übrigens der Ansicht, dass die Lösung des Jacobischen Umkehrproblems auf dem von Riemann in der Abhandlung, ,über die Theorie der Abelschen Functionen" (Bd.54 dieses Journals) vorgezeichneten Wege vollständig zu finden ist, wie ich dies im $\$$. 3 der vorliegenden Arbeit zu zeigen versucht habe. Eine einfache Anwendung des Abelschen Theorems führt in ähnlicher Weise, wie Abel das Additionstheorem für die elliptischen Integrale entwickelt hat*), und wie es später durch Herrn Weierstrass auf die hyperelliptischen Integrale ausgedehnt wurde $\left.{ }^{* *}\right)$, zur Lösung des Additionsproblems für die Integrale erster Gattung, eine Lösung, die sich mit Hülfe von Э-Functionen durch die unabhängigen Variablen des Umkehrproblems darstellen lässt. Genau dieselhen Betrachtungen führen auch zur Lösung des Additionsproblems für die zweite und dritte Gattung, wenn man gewisse Summen von Integralen zweiter und dritter Galtung, betrachtet als Functionen der Integralsummen erster Gattung, als Transcendenten zweiter und dritter Gattung einführt.

\section{\$. 1.}

Das Abelsche Theorem.

Da die im Folgenden aufzustellenden Sätze fast ausschliesslich auf der Anwendung des Abelschen Theorems beruhen, so sei es mir gestattet, dasselbe hier kurz auszusprechen in der Fassung, von welcher im Nachstehenden Gebrauch gemacht werden soll, und die im Wesentlichen übereinkommt mit der Form, in welcher Riemann dasselbe Theorem im $\$ .14$ der in der Einleitung citirten

*) Précis d'une théorie des fonctions elliptiques. Chapitre II. Bd. 4 dieses Journaks.

**) Bd. 52 dieses Journals.

Journal für Mathematik Bd. LXX. Heft 3. 
Abhandlung für die Integrale erster Gattung aufgestellt und für die übrigen algebraischen Integrale angedeutet hat.

Es sei $s$ eine algebraische Function von $z$ und mit $z$ durch die algebraische Gleichung

$$
\boldsymbol{F}\left(\begin{array}{c}
n, m \\
\boldsymbol{s}^{m}
\end{array}\right)=\mathbf{0}
$$

verbunden. Durch die Fläche $\boldsymbol{T}$ sei die Verzweigungsart der Function $s$ dargestellt. Es sei ferner $\zeta$ eine in der Fläche $\boldsymbol{T}$ einwerthige Function, die in $\nu$ Punkten dieser Fläche unendlich von der ersten Ordnung wird und demzufolge auch jeden beliebigen Werth in $\nu$ Punkten der Fläche $T$ annimmt. Eine solche Function kann durch $s$ und $z$ rational ausgedrückt werden *). Endlich bedeute $\omega$ irgend ein Integral einer rationalen Function von $s$ und $z$. Der Differentialquotient $\frac{\partial \omega}{\partial \zeta}$ ist dann eine algebraische Function von $\zeta$, welche für jeden Werth von $\zeta \nu$ Werthe hat, und wenn man diese Werthe mit $\frac{\partial \omega^{(h)}}{\partial \zeta}$ bezeichnet, so ist die Summe:

$$
\frac{\partial \omega^{(1)}}{\partial \zeta}+\frac{\partial \omega^{(2)}}{\partial \zeta}+\cdots+\frac{\partial \omega^{(v)}}{\partial \zeta}
$$

eine einwerthige und folglich rationale Function von $\zeta$, deren Integral durch rationale Functionen und Logarithmen ausgedrückt werden kann. Also:

Die Summe der Werthe des Integrals einer wie $\boldsymbol{T}$ verzweigten Function, bis zu solchen Punkten als oberen Grenzen erstreckt, in denen eine beliebige wie $T$ verzweigte Function $\zeta$ denselben $W^{\top}$ erth annimmt, ist durch rationale und logarithmische Functionen von $\zeta$ darstellbar.

Wendet man diesen Satz auf zwei verschiedene Werthe der Function $\zeta, \zeta$ und $\zeta_{0}$, an und bezeichnet die Punkte, in denen der Werth $\zeta$ stattindet, mit $x_{1}, x_{2}, \ldots x_{v}$, die Punkte, in denen der Werth $\zeta_{0}$ stattfindet, mit $x_{1}^{(0)}, x_{2}^{(0)}, \ldots x_{\nu}^{(0)}$, so fliessen daraus für die Integrale erster, zweiter und dritter Gattung die nachstehenden Formeln:

Bedeutet $\omega$ irgend ein Integral erster Gattung, so ist:

$$
\text { (1.) } \int_{x_{1}^{(0)}}^{x_{1}} d \omega+\int_{x_{2}^{(j)}}^{x_{2}} d \omega+\cdots+\int_{x_{\nu}^{(())}}^{x_{v}} d \omega=0 \text {. }
$$

Ist ferner $t_{(\varepsilon)}$ ein Integral zweiter Gattung, nämlich eine Function von der Eigenschaft, dass (falls $\varepsilon$ kein Verzweigungspunkt ist) $t_{(\varepsilon)}-\frac{1}{z-z_{\varepsilon}}$ im

*) Riemann 1. c. §. 8 . 
Punkte $\varepsilon$, dem der Werth $z_{\varepsilon}$ von $z$ entspricht, endlich bleibt, so ergiebt sich in gleicher Weise, wenn man mit $\eta$ und $\eta^{\prime}$ die Werthe der Function $\zeta$ und ihres Differentialquotienten im Unstetigkeitspunkt $\varepsilon$ bezeichnet:

$$
\int_{r_{1}^{(1)}}^{r_{1}} d t_{(\varepsilon)}+\int_{x_{2}^{(())}}^{x_{2}} d t_{(\varepsilon)}+\cdots+\int_{x_{v}^{(())}}^{r_{\nu}} d t_{(\varepsilon)}=\frac{\eta^{\prime}}{\zeta-\eta}-\frac{\eta^{\prime}}{\zeta_{0}-\eta},
$$

vorausgesetzt, dass in dem Punkte $\varepsilon$ nicht zwei oder mehrere einander gleiche Werthe von $\zeta$ zusammenfallen. (In diesem Ausnahmefall, der im Folgenden nicht ins Gewicht fällt, würde auf der rechten Seite der Gleichung (2.) ebenfalls $0 \mathrm{zu}$ stehen kommen.)

Endlich bezeichne $\pi_{\left(\varepsilon_{1} \varepsilon_{2}\right)}$ ein Integral dritter Gattung, also eine Function von der Beschaffenheit, dass $\pi_{\left(\varepsilon_{1} \varepsilon_{2}\right)}+\log \left(z-z_{\varepsilon_{1}}\right)$ und $\pi_{\left(\varepsilon_{1} \varepsilon_{2}\right)}-\log \left(z-z_{\varepsilon_{2}}\right)$ respective in den Punkten $\varepsilon_{1}, \varepsilon_{2}$ endlich bleiben (wieder vorausgesetzt, dass diese Punkte keine Verzweigungspunkte sind). Unter dieser Voraussetzung folgt aus dem Abelschen Satz, wenn $\eta_{1}, \eta_{2}$ die Werthe von $\zeta$ in den Punkten $\varepsilon_{1}$, $\varepsilon_{2}$ bedeuten:

$$
\int_{x_{1}^{0}}^{x_{1}} d \pi_{\left(\varepsilon_{1} \varepsilon_{2}\right)}+\int_{x_{2}^{0}}^{x_{2}} d \pi_{\left(\varepsilon_{1} \varepsilon_{2}\right)}+\cdots+\int_{x_{\nu}^{0}}^{x_{v}} d \pi_{\left(\varepsilon_{1} \varepsilon_{2}\right)}=\log \frac{\zeta-\eta_{2}}{\zeta-\eta_{1}}-\log \frac{\zeta_{0}-\eta_{2}}{\zeta_{0}-\eta_{1}} .
$$

Diese Formel bleibt auch dann noch richtig, wenn in dem Punkt $\varepsilon_{1}$ oder $\varepsilon_{2}$ oder in beiden zwei oder mehrere gleiche Werthe der Function $\zeta$ zusammenfallen; dagegen verschwindet hier die rechte Seite, sobald $\eta_{2}=\eta_{1}$ ist, d. h. sobald die Unstetigkeitspunkte solche sind, in welchen die Function $\zeta$ denselben Werth hat.

Die Formeln (1.), (2.), (3.) sind nur genau bis auf gewisse additive Constanten, welche aber lineare homogene Functionen mit ganzzahligen Coefficienten von den Periodicitätsmoduln der betreffenden Integrale sind; oder anders ausgedrückt: die Formeln (1.), (2.), (3.) sind nur richtig, wenn man die Integrationswege gehörig bestimmt, und zwar werden dieselben dabei durchans nicht nothwendig alle in der einfach zusammenhängenden Fläche $T^{\prime}$ verlaufen, sondern werden im Allgemeinen die Querschnitte überschreiten müssen. Nur so viel lässt sich im Allgemeinen schliessen, und das ist für die Folge von Wichtigkeit: die ganzzahligen Coefficienten der entsprechenden Periodicitätsmoduln in den erwähnten additiven Constanten werden für die Formeln (1.), (2.), (3.) und alle ähnlich gebildeten dieselben sein, sobald die Integrationswege zwischen denselben Grenzen dieselben sind. 


\section{\$. 2.}

Sätze über Integrale zweiter und dritter Gattung.

Ich stelle in diesem Paragraphen einige Sätze zusammen, von denen im Folgenden Gebrauch zu machen sein wird, die zum Theil von Riemann ausgesprochen sind, zum Theil in dem Werke über Abelsche Fnnctionen der Herren Clebsch und Gordan*) sich finden, und die ich daher nur kurz erwähnen werde, um ihnen diejenige Ausdrucksweise zu geben, die für den vorliegenden $Z$ weck die geeignete erscheint.

Die $(2 p+1)$ fach zusammenhängende Fläche $T$ werde durch die Querschnitte $a_{\nu} b_{\nu}$ in der Weise in eine einfach zusammenhängende Fläche $T^{\prime}$ verwandelt, wie es Riemann a. a. 0. \$. 20 auseinander gesetzt hat. Es sei $u_{\mu}$ eines der $\boldsymbol{p}$ Normalintegrale erster Gattung, dessen Periodicitätsmoduln an den Querschnitten $a_{v}$, mit Ausnahme von $a_{\mu}=0$, an $a_{\mu}=\pi i$ und an $b_{\mu}=a_{\mu \nu}$ sind, so dass $a_{\mu \nu}=a_{\nu \mu}$. Ferner mögen als Normalintergrale zweiter und dritter Gattung, $t_{\left(\varepsilon_{1}\right)}, \pi_{\left(\varepsilon_{1} \varepsilon_{2}\right)}$ solche betrachtet werden, deren Periodicitätsmoduln an den Querschnitten $a_{\mu}$ sämmtlich verschwinden, eine Form, die aus jedem Integral zweiter und dritter Gattung durch Hinzufügung eines geeigneten Integrals erster Gattung hergestellt werden kann.

Die gedachten Sätze fliessen nun alle sehr leicht aus einer und derselben Quelle, nämlich aus der Bestimmung der Werthe der folgenden Integrale:

$$
\text { (4.) } \int t_{(\varepsilon)} d u_{\mu}, \int \pi_{\left(\varepsilon_{1} \varepsilon_{2}\right)} d u_{\mu}, \int t_{(\varepsilon)} d \pi_{\left(\varepsilon_{1} \varepsilon_{2}\right)}, \int \pi_{\left(\varepsilon_{1} \varepsilon_{2}\right)} d \pi_{\left(\xi_{1} \xi_{2}\right)}
$$

in positiver Richtung um die ganze Begrenzung von $T^{\prime}$ erstreckt, welche Werthe gleich sein müssen den entsprechenden Integralen über beliebige Curven, welche die Unstetigkeitsstellen der unter dem Integralzeichen stehenden Functionen einschliessen.

Die beiden ersten Integrale ergeben auf diese Weise behandelt**) die Periodicitätsmoduln der Functionen $t_{(\varepsilon)}$ und $\pi_{\left(\varepsilon_{1} \varepsilon_{)}\right)}$an dem Querschnitt $a_{\mu}$, nämlich den von $t_{(\varepsilon)}$

und den von $\pi_{\left(\varepsilon_{1} \varepsilon_{2}\right)}$

$$
\text { (5.) } \quad-2\left(\frac{\partial u_{\mu}}{\partial z}\right)_{z=z_{\varepsilon}}
$$

(6.) $\quad 2\left(u_{\mu}^{\left(z_{\varepsilon_{2}}\right)}-u_{\mu}^{\left(z_{\varepsilon_{1}}\right)}\right)=\int_{\varepsilon_{1}}^{\varepsilon_{\varepsilon_{2}}} d u_{\mu}$.

*) Clebsch und Gordan, Theorie der Abelschen Functionen. Leipzig 1866. Fünfter Abschnitt.

**) Siehe Riemann 1. c. §. 20. 
Das dritte der obigen Integrale, in ähnlicher Weise behandelt, ergiebt die merkwürdige Formel:

$$
\text { (7.) } \int_{\varepsilon_{1}}^{\varepsilon_{2}} d t_{(\varepsilon)}=-\frac{\partial \boldsymbol{\pi}_{\left(\varepsilon_{1} \varepsilon_{2}\right)}^{\left(\boldsymbol{z}_{\varepsilon}\right)}}{\partial z_{\varepsilon}} \text {, }
$$

welche aussagt, dass das Normalintegral zweiter Gattung eine wie $T$ verzweigte, also algebraische Function seines Unstetigkeitspunktes ist, dass es also, wenn man die Grenzen als fest, den Unstetigkeitspunkt als veränderlich ansieht, rational durch die in seinem Unstetigkeitspunkte stattfindenden Werthe von $s$ und $z$ ausgedrückt werden kann. Das letzte Integral (4.), in gleicher Weise behandelt, führt zu dem Satz von der Vertauschung von Parameter und Argument für die Integrale dritter Gattung, nämlich zu der Formel:

$$
\text { (8.) } \int_{\varepsilon_{1}}^{\varepsilon_{2}} d \pi_{\left(\xi_{1} \xi_{2}\right)}=\int_{\xi_{1}}^{\xi_{2}} d \pi_{\left(\varepsilon_{1} \varepsilon_{2}\right)} \text {. }
$$

In den Formeln (6.), (7.), (8.) verlaufen die Integrationswege in der einfach zusammenhängenden Fläche $T^{\prime}$; bei anderer Bestimmung können noch Vielfache der Periodicitätsmoduln hinzutreten, und bei den Integralen dritter Gattung noch Vielfache von $2 \pi i$.

\section{\$. 3.}

Das Umkehrproblem.

Es sollen nun, um später Unklarheiten zu vermeiden, die Formeln aufgestellt werden, die sich, mit Hülfe des Abelschen Theorems, unmittelbar aus Riemanns Resultaten ergeben; und die das Umkehrproblem in der Jacobischen Fassung lösen, Formeln, die im Wesentlichen übereinstimmen mit den von den Herren Clebsch und Gordan*) auf anderem Wege entwickelten. Ich bediene mich dabei, um eine Grössenreihe wie

$$
v_{1}, v_{2}, \ldots v_{m}
$$

abgekürzt darzustellen, der von Riemann in der Abhandlung ,über das Verschwinden der 9 -Functionen ${ }^{* * *}$ ) gebrauchten sehr zweckmässigen Bezeichnung:

$$
\left(\begin{array}{l}
m \\
h \\
1
\end{array}\left(v_{h}\right)\right),
$$

wobei $h$ gewissermassen wie der Index bei einem Summenzeichen, 1 und $m$ wie die Grenzen eines solchen zu betrachten sind. Bezeichnet man nun mit

*) 1. c. Sechster Abschnitt.

**) Bd. 65 dieses Journals. 
$v_{1}, v_{2} \ldots v_{p}$ ein beliebiges Grössensystem (unabhängige Variable), so lassen sich, wie Riemann nachgewiesen hat ${ }^{*}$, immer, und im Allgemeinen, d. h. wenn zwischen den Grössen $v_{1}, v_{2} \ldots v_{p}$ nicht gewisse Bedingungen bestehen, nur auf eine Weise die Punkte $x_{1}, x_{2} \ldots x_{p}$ in der Fläche $T$ so bestimmen, dass die Gleichungen erfüllt sind:

$$
\text { (9.) } \quad v_{\varrho}=\int_{c_{1}}^{x_{1}} d u_{\varrho}+\int_{c_{2}}^{x_{2}} d u_{Q}+\cdots+\int_{c_{p}}^{x_{p}} d u_{\rho},
$$

vorausgesetzt, dass die Integrationswege gehörig bestimmt werden. Es bedeuten dabei $c_{1}, c_{2} \ldots c_{p}$ beliebige, ein für allemal als fest angenommene Punkte, welche möglicherweise auch alle oder theilweise in einen zusammenfallen können. Ich hebe gleich hier hervor, dass ich immer voraussetze, dass in den $p$ in (9.) enthaltenen Gleichungen zu derselben oberen Grenze auch immer dieselbe untere Grenze gehöre. Bei der von Riemann für die Argumente der $\boldsymbol{9}$-Function festgesetzten Bestimmungsart der unteren Grenzen sind gerade umgekehrt für die verschiedenen Integrale erster Gattung die unteren Grenzen wesentlich verschieden, dagegen für dasselbe Integral, auch wenn die oberen Grenzen verschieden sind, immer dieselbe, ein Umstand, der bei der Anwendung der Formeln von Riemann auf unsern Fall wohl zu beachten ist.

Das Jacobische Umkehrproblem besteht nun darin, wenn die Grössen $v_{1}, v_{2} \ldots v_{p}$ als beliebig gegeben betrachtet werden, die Punkte $x_{1}, x_{2} \ldots x_{p}$ zu ermitteln, und um diese Aufgabe zu lösen bieten die Riemannschen Untersuchungen zwei verschiedene Wege dar.

Der eine dieser Wege besteht darin, dass man direct darauf ausgeht, mit Hülfe von 9 -Funclionen eine rationale Function von $s$ und $z$ zu bilden, welche gerade in den Punkten $x_{1}, x_{2} \ldots x_{p}$ und in keinen andern, in diesen aber mindestens von der zweiten Ordnung unendlich klein wird, und dazu sind in \$. 27 der mehrfach citirten Riemannschen Abhandlung die Mittel vollständig gegeben. Der zweite Weg den ich hier in der Kürze darlegen will, löst das Problem durch Vermittlung von Integralen dritter Gattung in ähnlicher Weise, wie es von den Herren Clebsch und Gordan durchgeführt ist.

Betrachtet man eine Summe von Normalintegralen dritter Gattung:

$$
\int_{c_{1}}^{x_{1}} d \pi_{\left(\varepsilon_{1} \varepsilon_{2}\right)}+\int_{c_{2}}^{x_{2}} d \pi_{\left(\varepsilon_{1} \varepsilon_{2}\right)}+\cdots+\int_{c_{p}}^{x_{p}} d \pi_{\left(\varepsilon_{1} \varepsilon_{2}\right)}
$$

*) Theorie der Abelschen Fnnctionen §̧. 24. Eingehender, rücksichtlich der Ausnahmefälle in der erwähnten Abhandlung „über das Verschwinden der $\vartheta$-Functionen“. 
so kann diese Summe, vorausgesetzt dass man die Integrationswege in derselben $W^{\prime}$ eise bestimmt, wie in den Gleichungen (9.), als Function der Grössen $v_{1}, v_{2} \ldots v_{p}$ angesehen werden, welche bis auf Vielfache von $2 \pi i$ völlig eindeutig ist. So betrachtet möge diese Function bezeichnet werden durch:

$$
\boldsymbol{P}_{\varepsilon_{1} \varepsilon_{2}}\left(\boldsymbol{v}_{1}, \boldsymbol{v}_{2} \ldots \boldsymbol{v}_{p}\right) \text {. }
$$

Diese Summe findet sich bei Riemann (1. c. \$. 25.) durch $\vartheta$-Functionen ausgedrückt. Bezeichnet man diejenigen Punkte, welche nach Riemanns Bestimmungsweise den unteren Grenzen der Integrale erster Gattung entsprechen, mit $\gamma_{1}, \gamma_{2} \ldots \gamma_{n}$, so ergiebt sich aus den erwähnten Formeln ohne Weiteres:

(11.) $P_{\varepsilon_{1} \varepsilon_{2}}\left(v_{1}, v_{2} \ldots v_{p}\right)=\log \frac{\gamma_{h}}{\vartheta\left(\underset{h}{h}\left(\int_{\gamma_{h}}^{\varepsilon_{1}} d u_{h}-v_{h}+k_{h}\right)\right) \vartheta\left(\underset{h}{h}\left(\int_{\gamma_{h}}^{\varepsilon_{9}} d u_{h}+k_{h}\right)\right)}$,

$$
\frac{\vartheta\left(\begin{array}{l}
p \\
h
\end{array}\left(\int_{\gamma_{h}}^{\varepsilon_{2}} d u_{h}-v_{h}+k_{h}\right)\right) \vartheta\left(\begin{array}{l}
p \\
h
\end{array}\left(\int_{\gamma_{h}}^{\varepsilon_{1}} d u_{h}+k_{h}\right)\right)}{\vartheta\left(\begin{array}{l}
p \\
h \\
1
\end{array}\left(\int_{\gamma_{h}}^{\varepsilon_{1}} d u_{h}-v_{h}+k_{h}\right)\right) \vartheta\left(\begin{array}{l}
p \\
h
\end{array}\left(\int_{\gamma_{h}}^{\varepsilon_{3}} d u_{h}+k_{h}\right)\right)},
$$

worin die Constanten $k_{h}$ die Bedeutung haben:

$$
\text { (12.) } \left.\quad k_{h}=\int_{c_{1}}^{\gamma_{h}} d u_{h}+\int_{c_{2}}^{\gamma_{h}} d u_{h}+\cdots+\int_{c_{p}}^{\gamma_{h}} d u_{h}^{*}\right) \text {. }
$$

Nimmt man nun eine beliebige rationale Function $\sigma$ von $s$ und $z$ an, die in

*) Die Argumente der $\vartheta$-Functionen lassen sich ohne Kenntniss der Punkte $\gamma_{h}$ vollständig durch die $v_{h}$ ausdrücken. Versteht man nämlich unter $c_{0}$ eine beliebige, allen Integralen erster Gattung gemeinschaftliche untere Grenze, so kommt es, um in (11.) die Argumente der $\vartheta$-Functionen vollständig ausdrücken zu können, nur auf die Kenntniss von

$$
\int_{\gamma_{h}}^{c_{0}} d u_{h}+k_{h}
$$

an. Setzt man aber für die Argumente der $\vartheta$-Functionen:

$$
\int_{c_{0}}^{z, s} d u_{h}-\sum_{i=1}^{i=p} \int_{c_{0}}^{x_{i}} d u_{h}+k_{h}^{\prime}
$$

wenn die $\vartheta$-Function in den Punkten $x_{i}$ verschwinden soll, so lassen sich nach Riemann (über das Verschwinden der $\vartheta$-Functionen) die Constanten $k_{h}^{\prime}$ vollständig bestimmen. Dies vorausgesetzt folgt aus der Gleichung:

$$
\begin{gathered}
\int_{\gamma_{h}}^{z, s} d u_{h}-\sum_{i=1}^{i=p} \int_{c_{i}}^{x_{i}} d u_{h}+k_{h}=\int_{c_{0}}^{z, s} d u_{h}-\sum_{i=1}^{i=p} \int_{c_{0}}^{x_{i}} d u_{h}+k_{h}^{\prime}, \\
\int_{\gamma_{h}}^{c_{0}} d u_{h}+k_{h}=\sum_{i=1}^{i=p} \int_{c_{i}}^{c_{0}} d u_{h}+k_{h}^{\prime},
\end{gathered}
$$

wo auf der rechten Seite nur bekannte Grössen stehen. 
“ Punkten $\dot{\xi}_{1}^{(())}, \dot{\xi}_{2}^{(())} \ldots \dot{\xi}_{\mu}^{(())}$unendlich von der ersten Ordnung wird, und daher auch jeden andern Werth $\sigma$ in $\mu$ Punkten $\xi_{1}, \xi_{2} \ldots \xi_{\mu}$ annimmt, so kann die Aufgabe des Umkehrproblems so gestellt werden, dass die ganze rationale Function $p^{\text {ten }}$ Grades von $\sigma$ :

$$
\psi(\sigma)=\left(\sigma-\sigma_{1}\right)\left(\sigma-\sigma_{2}\right) \ldots\left(\sigma-\sigma_{p}\right)=\sigma^{p}+M_{1} \sigma^{p-1}+\cdots+M_{p-1} \sigma+M_{p}
$$

bestimmt werden soll, deren Wurzeln $\sigma_{1}, \sigma_{2} \ldots \sigma_{p}$ die $p$ Werthe der Function $\sigma$ in den Punkten $x_{1}, x_{2} \ldots x_{p}$ sind. Bezeichnet man aber mit $\sigma_{1}^{(1)}, \sigma_{2}^{(U)} \ldots \sigma_{p}^{(1)}$ die Werthe derselben Functionen in den festen Punkten $c_{1}, c_{2} \ldots c_{p}$, so ist nach dem Abelschen Theorem:

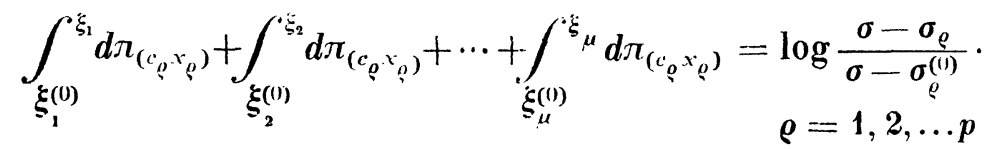

Addirt man diese Gleichungen für die verschiedenen Werthe von $\varrho$ und wendet den Satz von der Vertauschung des Arguments und Parameters an, so folgt:

$$
\log \frac{\left(\sigma-\sigma_{1}\right)\left(\sigma-\sigma_{2}\right) \ldots\left(\sigma-\sigma_{p}\right)}{\left(\sigma-\sigma_{1}^{(1)}\right)\left(\sigma-\sigma_{2}^{(1)}\right) \ldots\left(\sigma-\sigma_{p}^{(())}\right)}=\sum_{i=1}^{i=\mu} P_{\xi_{i}^{(1)} \xi_{i}}\left(v_{1}, v_{2} \ldots v_{p}\right)
$$

und daraus, wenn man schliesslich noch $\left(\sigma-\sigma_{1}^{(())}\right)\left(\sigma-\sigma_{2}^{(())}\right) \ldots\left(\sigma-\sigma_{p}^{((1)}\right)$ mit $\psi^{(())}(\sigma)$ bezeichnet:

$$
\begin{aligned}
& \vartheta\left({ }_{1}^{p}\left(\int \xi^{\xi_{i}} d u_{h}-v_{h}+k_{h}\right)\right) \vartheta\left(\begin{array}{l}
p \\
h
\end{array}\left(\int^{\xi_{i}^{(1)}} d u_{h}+k_{h}\right)\right) \\
& \frac{\psi(\sigma)}{\psi^{(0)}(\sigma)}=\prod_{i=1}^{i=\mu} \frac{\gamma_{h}}{\vartheta\left(\underset{1}{\boldsymbol{p}}\left(\int_{\gamma_{h}}^{\xi_{i}^{(())}} d u_{h}-v_{h}+k_{h}\right)\right) \vartheta\left({ }_{h}^{p}\left(\int_{\gamma_{h}}^{p} \xi_{i} d u_{h}+k_{h}\right)\right)} .
\end{aligned}
$$

Dieser Ausdruck könnte möglicher Weise noch einen von $\sigma$ unabhängigen Factor enthalten; man sieht aber, dass dieser Factor $=1$ sein muss, wenn man die Werthe von $\sigma$ nicht weiter ausdehnt, als dass ohne Ueberschreitung der Querschnitte sämmtliche $\xi_{i}$ in die entsprechenden $\xi_{i}^{(())}$übergeführt werden können; denn dann wird für $\sigma=\infty$ die Formel (14.) identisch. Die Coefficienten $M_{1}, M_{2} \ldots M_{p}$ von $\psi$ können bestimmt werden, wenn man die Gleichung (14.) für $p$ verschiedene Werthe von $\sigma$ aufstellt. Die Formel (14.) stimmt mit der der Herren Clebsch und Gordan*) im Wesentlichen überein.

*) 1. c. $\$ .57$. 
\$. 4.

Das Additionstheorem für die erste Gattung.

Bezeichnet man, wie oben durch $v_{1}, v_{2}, \ldots, v_{p}$, ein zweites beliebiges Grössensystem mit $w_{1}, w_{2}, \ldots, w_{p}$, so lassen sich, wie aus der Gleichung (9.) die Punkte $x_{1}, x_{2}, \ldots, x_{p}$, so aus :

$$
\begin{gathered}
\text { (15.) } \quad w_{\rho}=\int_{c_{1}}^{y_{1}} d u_{Q}+\int_{c_{9}}^{y_{2}} d u_{Q}+\cdots+\int_{c_{p}}^{\gamma_{p}} d u_{\rho} \\
\varrho=1,2 \ldots p
\end{gathered}
$$

die Punkte $y_{1}, y_{2}, \ldots, y_{p}$ in unzweideutiger Weise bestimmen.

Die Aufgabe des Addilionstheorems ist nun folgende:

Setzt man die Grössen $v_{1}, v_{2}, \ldots, v_{p} ; w_{1}, w_{2}, \ldots, v_{p}$ als gegeben und die Punkte $x_{1}, x_{2}, \ldots, x_{p} ; y_{1}, y_{2}, \ldots, y_{p}$ als daraus bestimmt voraus, so sollen aus diesen Punkten $x_{1}, x_{2}, \ldots, x_{p} ; y_{1}, y_{2}, \ldots, y_{p}$ die neuen Punkte $z_{1}, z_{2}, \ldots, z_{p}$ so bestimmt werden, dass den Gleichungen Genüge geschieht:

$$
\begin{gathered}
\text { (16.) } \boldsymbol{v}_{\varrho}+w_{\varrho}=\int_{c_{1}}^{z_{1}} d u_{\varrho}+\int_{c_{3}}^{z_{2}} d u_{\rho}+\cdots+\int_{c_{p}}^{z_{p}} d u_{\varrho} . \\
\varrho=1,2 \ldots p
\end{gathered}
$$

Die Punkte $z_{1}, z_{2}, \ldots, z_{p}$ sind dadurch im Allgemeinen ebenfalls völlig bestimm! und können aus den $x_{i}, y_{i}$ auf algebraischem Wege gefunden werden.

Aus den Gleichungen (9.), (15.), (16.) ergeben sich durch Elimination der $v_{Q}$ und $w_{o}$ für die $z_{i}$ die Bedingungen:

$$
\begin{gathered}
0=\int_{x_{1}}^{z_{1}} d u_{\rho}+\int_{x_{1}}^{z_{2}} d u_{\rho}+\cdots+\int_{\substack{x_{p} \\
\rho=1,2 \ldots p}}^{z_{p}} d u_{\rho}+\int_{y_{1}}^{c_{1}} d u_{\rho}+\int_{y_{1}}^{c_{2}} d u_{\rho}+\cdots+\int_{y_{p}}^{c_{p}} d u_{\rho}, \\
\rho=1,2 \ldots p
\end{gathered}
$$

und zur Lōsung dieses Systems von Gleichungen liefert das Abelsche Theorem die Mittel.

Nach einem von Riemann bewiesenen Satze *) lässt sich eine rationale Function $\zeta$ von $s$ und $z$ bestimmen, welche in den $p$ Punkten $x_{1}, x_{2}, \ldots, x_{p}$; $y_{1}, y_{2}, \ldots, y_{p}$ unendlich von der ersten Ordnung wird, und welche noch $p+1$ willkürliche Constanten enthält, welche linear und homogen in ihr enthalten sind. Von diesen $p+1$ Constanten lassen sich $p$ so bestimmen, dass $\zeta$ einen beliebigen Werth, z. B. den Werth 0 in den $p$ Punkten $c_{1}, c_{2}, \ldots, c_{p}$ annimmt. Ist dieser Werth von $\zeta$ gerade 0 , so ist $\zeta$ dadurch bis auf einen von $s$ und

*) l. c. $\S .5, \S .8$.

Journal für Mathematik Bd. LXX. Heft 3. 
unabhängigen Factor hestimmt und verschwindet ausser in den Punkten $c$ noch in $p$ anderen Punkten, welche in Folge des Abelschen Theorems (Formel (1.) ๑. 1) mit den Punkten $z_{1}, z_{2}, \ldots, z_{p}$ zusammenfallen. Ist also die Function $\zeta$ gefunden, so erhält man die $z_{1}, z_{2}, \ldots, z_{p}$ auf algebraischem Wege.

Es ist nun nachzuweisen, dass diese Function $\zeta$ auch rational ausgedrückt werden kann durch die in den Punkten $x_{i} y_{i}$ slattfindenden Werthe von $s$ und $z: s_{x_{i}} z_{x_{i}}, s_{y_{i}} z_{y_{i}}$.

Man kann nach Riemann (1. c. \$. 5.) die Function $\zeta$ dadurch bilden, dass man in dem Ausdruck

$$
\zeta=\alpha_{1} t_{x_{1}}+\alpha_{2} t_{x_{3}}+\cdots+\alpha_{p} t_{x_{p}}+\beta_{1} t_{y_{1}}+\beta_{2} t_{y_{2}}+\cdots+\beta_{p} t_{y_{p}}+\text { Const. }
$$

worin die $t$ wie oben Normalintegrale zweiter Gattung bedeuten, die Constanten $\alpha \beta$ so bestimmt, dass die Periodicitätsmoduln an den Querschnilten $b_{\nu}$ sämmtlich 0 werden.

Daraus erhält man ein System von $p$ linearen homogenen Gleichungen für die $\alpha \beta$ als Unbeliannte, deren Coefficienten nach $\$$. 2 (5.) rationale Functionen der $s_{x_{i}} z_{x_{i}}, s_{y_{i}} z_{y_{i}}$ sind, so dass $p$ von den Constanten $\alpha \beta$ als lineare homogene Functionen der übrigen bestimmt werden, mit Coefficienten, welche in Bezug auf $s_{x_{i}} z_{x_{i}}, s_{y_{i}} z_{y_{i}}$ rational sind. Ausserdem aber kommen nach $\$ .2$ (7.) die $s_{x_{i}} z_{x_{i}}, s_{y_{i}} z_{y_{i}}$, da die additive Constante bis jetzt noch unbestinmt ist, in $\zeta$ auch nur ralional vor. Ausser den $p+1$ bis jetzt noch übrig bleibenden willkürlichen Constanten enthält also $\zeta$ die $s_{x_{i}} z_{x_{i}}, s_{y_{i}} z_{y_{i}}$ nur rational. Die Verhälınisse der $p+1$ noch übrig bleibenden Constanten werden aber durch lineare Gleichungen bestimmt, die man daraus erhält, dass $\zeta$ in den $p$ Punkten $c_{1}, c_{2}, \ldots, c_{p}$ verschwinden soll, wodurch also keine Irrationalität eingeschleppt werden kann. Demnach kann $\zeta$ rational durch $s, z ; s_{x_{i}}, z_{x_{i}} ; s_{y_{i}}, z_{y_{i}}$ ausgedrückt werden. Die Punkte $c_{i}$ betrachten wir als numerisch gegeben; es ist übrigens auch $\zeta$ durch $s_{c_{i}} z_{c_{i}}$ ralional ausdrückbar, wenn auch die $c_{i}$ als veränderlich angesehen werden.

Nach Analogie der Jacobischen Fassung des Umkehrproblems lässt sich dem Additionsproblem eine elegantere Form geben: Bedeutet $\sigma$ irgend eine rationale Function von $s$ und $z$ (z. B. auch $s$ oder $z$ selbst), so wird diese in den Punkten $z_{1}, z_{2}, \ldots, z_{p}$ die Werthe $\sigma_{z_{1}}, \sigma_{z_{2}}, \ldots, \sigma_{z_{p}}$ annehmen. Es sollen die Coefficienten der algebraischen Gleichung:

(18.) $\psi(\sigma)=\left(\sigma-\sigma_{z_{1}}\right)\left(\sigma-\sigma_{z_{3}}\right) \ldots\left(\sigma-\sigma_{z_{p}}\right)=\sigma^{p}+M_{1} \sigma^{p-1}+\cdots+M_{p-1} \sigma+M_{p}=0$, durch die Punkte $x_{i} y_{i}$ ausgedrückt, gefunden werden. 
Aus der Function $\zeta$ ergiebt sich die Function $\psi(\sigma)$ und zwar rational durch $z_{x_{i}} s_{x_{i}}, z_{y_{i}} s_{y_{i}}$ ausgedrückt:

Wird $\sigma$ in $u$ Punkten unendlich von der ersten Ordnung, so nimmt es auch jeden beliebigen Werth in $\mu$ Punkten an, und es entsprechen jedem Werth von $\sigma$ demnach $\mu$ Werthe von $\zeta$, so dass $\zeta$ als eine $\mu$-werthige Function von $\sigma$ angesehen werden kann.

Bezeichnet man die einem und demselben Werthe von $\sigma$ zukommenden Werthe von $\zeta$ mit $\zeta^{(1)}, \zeta^{(2)}, \ldots, \zeta^{(\mu)}$, so ist das Product:

$$
\zeta^{(1)} \zeta^{(2)} \ldots \zeta^{(\mu)}=\chi(\sigma)
$$

eine einwerlhige und folglich rationale Funclion von $\sigma$, welche unendlich von der ersten Ordnung wird für $\sigma=\sigma_{x_{1}} \sigma=\sigma_{x_{2}} \ldots \sigma=\sigma_{x_{p}}, \sigma=\sigma_{y_{1}} \sigma=\sigma_{y_{2}} \ldots \sigma=\sigma_{y_{p}}$, und welche unendlich klein von der ersten Ordnung wird für $\sigma=\sigma_{c_{1}} \quad \sigma=\sigma_{c_{2}} \ldots$ $\sigma=\sigma_{c_{p}}$. Es ist daher

$$
\frac{\chi(\sigma)\left(\sigma-\sigma_{x_{1}}\right)\left(\sigma-\sigma_{x_{s}}\right) \ldots\left(\sigma-\sigma_{r_{p}}\right)\left(\sigma-\sigma_{y_{1}}\right)\left(\sigma-\sigma_{y_{2}}\right) \ldots\left(\sigma-\sigma_{y_{p}}\right)}{\left(\sigma-\sigma_{c_{1}}\right)\left(\sigma-\sigma_{c_{2}}\right) \ldots\left(\sigma-\sigma_{c_{p}}\right)}
$$

eine ganze rationale Function von $\sigma$, welche nur für $\sigma=\sigma_{z_{1}} \sigma=\sigma_{z_{2}} \ldots \sigma=\sigma_{z_{p}}$ verschwindet, und mithin bis auf einen von $\sigma$ unabhängigen Factor mit der Function $\psi(\sigma)$ übereinstimmt. Die Operationen, die zur Bildung dieser Function vorgenommen sind, beruhen nur auf Division rationaler Functionen und können daher keine Irrationalität einführen.

Der Addition gegenüber steht die Subtraction, welche eine ganz ăhnliche Behandlung gestattet, so dass eine kurze Andeutung genügen wird.

Die Aufgabe ist die, aus den als bekannt vorausgesetzten Punkten $x_{i}, y_{i}$ die Punkte $z_{i}^{\prime} z u$ bestimmen, die der Bedingung genũgen:

$$
\begin{gathered}
v_{Q}-w_{\rho}=\int_{c_{1}}^{z_{1}^{\prime}} d u_{\rho}+\int_{c_{2}}^{z_{2}^{\prime}} d u_{\rho}+\cdots+\int_{c_{p}}^{z_{p}^{\prime}} d u_{\rho} . \\
\rho=1,2, \ldots p
\end{gathered}
$$

Diese Punkte mũssen in Folge der Formeln (9.), (15.) die Gleichungen orfüllen :

(20.) $0=\int_{c_{1}}^{z_{1}^{\prime}} d u_{\rho}+\int_{c_{2}}^{z_{2}^{\prime}} d u_{\rho}+\cdots+\int_{e_{p}}^{x_{p}^{\prime}} d u_{\rho}+\int_{x_{1}}^{y_{1}} d u_{\rho}+\int_{x_{1}}^{y_{2}} d u_{\rho}+\cdots+\int_{x_{p}}^{y_{p}} d u_{\rho}$. $\rho=1,2, \ldots p$

Man kann nun wie oben eine Function $\zeta^{\prime}$ von $s$ und $z$ bestimmen, welche in den Punkten $x_{1}, x_{2}, \ldots, x_{p}, c_{1}, c_{2}, \ldots, c_{p}$ unendlich von der ersten Ordnung, in $y_{1}, y_{2}, \ldots, y_{p}$ unendlich klein von der ersten Ordnung wird, welche dadurch 
bis auf einen von $s$ und $z$ unabhängigen Factor bestimmt ist. Auch $\zeta^{\prime}$ kann rational durch $s_{x_{i}} z_{x_{i}}, s_{y_{i}} z_{y_{i}}$ ausgedrückt werden, und diejenigen $p$ Punkte, in welchen dieselbe ausser den Punkten $y_{i}$ noch verschwindet, sind die gesuchten Punkte $z_{1}^{\prime}, z_{2}^{\prime}, \ldots, z_{p}^{\prime}$.

Man kann ebenso wie oben eine algebraische Gleichung herstellen, deren Wurzeln die $p$ Werthe einer rationalen Function von $s$ und $z$ in den $p$ Punkten $z_{i}^{\prime}$ ergeben.

S. 5 .

Lösung des Additionsproblems durch $\vartheta$-Functionen.

Die oben mit $\psi(\sigma)$ bezeichnete Function, ebenso wie die Function $\zeta$ sind in Bezug auf die Punkte $x_{1}, x_{2}, \ldots, x_{p}$ und $y_{1}, y_{2}, \ldots, y_{p}$, einzeln genommen, symmetrisch. Es muss daher möglich sein, diese beiden Functionen mit Hülfe von $\boldsymbol{\vartheta}$-Functionen durch die Grössen $\boldsymbol{v}_{i} w_{i}$ auszudrücken. Ein solcher Ausdruck, der die Grössen $v_{i}$ und $w_{i}$ gemischt enthält, lässt sich ohne Weiteres angeben. In Folge der Definition der Grössen $k_{h}(\$ .3(12)$.$) ver-$ schwindet nämlich die Function:

$$
\vartheta\left(\underset{1}{h}\left(\int_{\gamma_{h}}^{z, s} d u_{h}+k_{h}\right)\right)
$$

als Function von $z, s$ betrachtet, in den Punkten $c_{1}, c_{2}, \ldots, c_{p}$; ebenso die beiden Functionen

$$
\vartheta\left({ }_{1}^{p}\left(\int_{\gamma_{h}}^{z, s} d u_{h}-v_{h}+k_{h}\right)\right), \quad \vartheta\left(\underset{1}{h}\left(\int_{\gamma_{h}}^{z, s, s} d u_{h}-w_{h}+k_{h}\right)\right)
$$

respective in den Punkten $x_{1}, x_{2}, \ldots, x_{p}$ und $y_{1}, y_{2}, \ldots, y_{p}$ und endlich die Function:

$$
\vartheta\left(\underset{1}{h}\left(\int_{\gamma_{h}}^{z, s} d u_{h}-\left(\boldsymbol{v}_{h}+w_{h}\right)+k_{h}\right)\right)
$$

in den Punkten $z_{1}, z_{2}, \ldots, z_{p}$, und daraus ergiebt sich, wenn man von einem constanten, d. h. von $s, z$ unabhängigen Factor absieht:

(21.)

$$
\frac{\vartheta\left({ }_{1}^{p}\left(\int_{\gamma_{h}}^{x, s} d u_{h}-\left(v_{h}+v_{h}\right)+k_{h}\right)\right) \vartheta\left({ }_{1}^{p}\left(\int_{\gamma_{h}}^{z, s} d u_{h}+k_{h}\right)\right)}{\vartheta\left(\underset{1}{h}\left(\int_{\gamma_{h}}^{z, s} d u_{h}-v_{h}+k_{h}\right)\right) \vartheta\left(\begin{array}{l}
p \\
h
\end{array}\left(\int_{\gamma_{h}}^{z, s} d u_{h}-w_{h}+k_{h}\right)\right)},
$$


da der Ausdruck auf der rech'ell Seite in Folge der Fundamentaleigenschaft der 9 -Function an den Querschnitten keine Aenderung erleidet.

Es ist aber diese Formel nicht als eine Lösung des Additionsproblems zu betrachten, da eben darin noch die Argumente $v_{h}+w_{h}$ enthalten sind. Die sich zunächst bietende Aufgabe ist die, die Function $\zeta$ in der Weise auszudrücken, dass nur $\boldsymbol{\vartheta}-$ Functionen vorkommen, die $\boldsymbol{v}_{h}$ allein und $\boldsymbol{w}_{h}$ allein enthalten. Einen ganz einfachen Ausdruck wird man dabei nicht erwarten dürfen, da schon die Function $\zeta$ die $x_{1}, x_{2}, \ldots, x_{p}, y_{1}, y_{2}, \ldots, y_{p}$ in einer Weise gemischt enthält, die eine einfache Separation nicht gestattet. Man kann aber, was aus der ursprünglichen Definition der Function $\zeta$ nicht unmiltelbar ersichtlich ist, die Separation durch Auflösung eines Systems von $p$ linearen Gleichungen bewerkstelligen, und so, wenn man will, $\zeta$ durch Determinanten darstellen. $\mathrm{Zu}$ dieser Darstellung der Function $\zeta$ führt die Bemerkung, dass dieselbe sich stets linear zusammenselzen lässt aus $p$ ähnlichen Functionen, welche in denselben Punkten unendlich, aber in $p$ beliebigen anderen Punkten Null werden. Bezeichnet man $p$ solcher Functionen mit $\zeta_{1}, \zeta_{2}, \ldots, \zeta_{p}$, so erhält man die Function $\zeta$ dadurch, dass man die Verhältnisse der $p+1$ Constanten $a_{10}, a_{1}, \ldots, a_{p}$ so bestimmt, dass

$$
\text { (22.) } \zeta=a_{0}+a_{1} \zeta_{1}+a_{2} \zeta_{2}+\cdots+a_{p} \zeta_{p}
$$

in den $p$ Punkten $c_{1}, c_{2}, \ldots, c_{p}$ verschwindet. Solche Functionen $\zeta_{1}, \zeta_{2}, \ldots, \zeta_{p}$ lassen sich aber leicht bilden durch $\vartheta$-Functionen, die respective nur von den $v_{1}, v_{2}, \ldots, v_{p}$ oder $w_{1}, w_{2}, \ldots, w_{p}$ abhängen.

Selzt man nämlich :

(23.)

$$
\zeta_{\varrho}=\frac{\vartheta\left(\begin{array}{l}
p \\
h
\end{array}\left(\int_{\gamma_{h}}^{z, s} d u_{h}-v_{h}+g_{h}^{(o)}+k_{h}\right)\right) \vartheta\left(\begin{array}{l}
p \\
h \\
1
\end{array}\left(\int_{\gamma_{h}}^{z, s} d u_{h}-w_{h}-g_{h}^{(o)}+k_{h}\right)\right)}{\vartheta\left(\begin{array}{l}
p \\
h
\end{array}\left(\int_{\gamma_{h}}^{z, s} d u_{h}-v_{h}+k_{h}\right)\right) \vartheta\left({ }_{1}^{p}\left(\int_{\gamma_{h}}^{z, s} d u_{h}-w_{h}+k_{h}\right)\right)},
$$

so hat dieser Ausdruck, wenn die $g_{i}^{(\rho)}, g_{2}^{(\rho)}, \ldots, g_{p}^{(!)}$ganz beliebige Grössen bedeuten, alle von den Functionen $\zeta_{\varrho}$ verlangten Eigenschaften, da der Quotient auf der rechten Seite, wenn $z, s$ irgend einen der Querschnitte überschreitet, keine Aenderung erleídet. Von den Werthen der Grössen $g_{h}^{(!)}$hängen die Punkte $a b$, in welchen die Function $\zeta_{\varrho}$ verschwindet, und man kann die Grössen $g_{h}^{(\rho)}$ immer so bestimmen, dass $\zeta_{\varrho}$ in $p$ beliebig gewählten Punkten verschwindet, so dass durch (23.) die allgemeinste Function $\zeta_{\rho}$ dargestellt ist. Man hat 
daher, um die $p$ Functionen $\zeta_{1}, \zeta_{2}, \ldots, \zeta_{p}$ zu erhalten, $p$ verschiedene aber beliebige Grössensysteme $g_{1}^{(o)}, g_{2}^{(\rho)}, \ldots, g_{p}^{(\rho)}$ zu nehmen, so dass nach dem oben Bemerkten die Function $\zeta$ rational durch solche $\vartheta-F u n c t i o n e n$ darstellbar ist.

Man kann z. B. die Grössen $g_{h}^{(o)}$ nach dem Schema bestimmen:

$$
\begin{aligned}
& \begin{array}{llll}
\pi i & 0 & \ldots & 0
\end{array} \\
& 0 \quad \frac{\pi i}{2} \ldots 0 \\
& 0 \quad 0 \quad \ldots \frac{\pi i}{2} .
\end{aligned}
$$

Berúcksichtigt man dann, dass die Function $\zeta$ um $\pi i$ periodisch ist, und setzt zur Abkürzung :

$$
\left\{\begin{array}{l}
\vartheta\left(\omega_{1}+\frac{\pi i}{2}, \omega_{2}, \ldots, \omega_{p}\right)=\vartheta_{1}\left(\omega_{1}, \omega_{2}, \ldots, \omega_{p}\right), \\
\vartheta\left(\omega_{1}, \omega_{2}+\frac{\pi i}{2}, \ldots, \omega_{p}\right)=\vartheta_{2}\left(\omega_{1}, \omega_{2}, \ldots, \omega_{p}\right), \\
\cdot \cdot \cdot \cdot \cdot \cdot \cdot . \cdot . \cdot . \cdot . \\
\vartheta\left(\omega_{1}, \omega_{2}, \ldots, \omega_{p}+\frac{\pi i}{2}\right)=\vartheta_{p}\left(\omega_{1}, \omega_{2}, \ldots, \omega_{p}\right)
\end{array}\right.
$$

so orhălt man für $\zeta_{\rho}$ den Ausdruck:

$$
\frac{\vartheta_{\varphi}\left(\begin{array}{l}
p \\
h
\end{array}\left(\int_{\gamma_{h}}^{z, s} d u_{h}-v_{h}+k_{h}\right)\right) \vartheta_{\varphi}\left(\begin{array}{l}
p \\
h
\end{array}\left(\int_{\gamma_{h}}^{z, s} d u_{h}-w_{h}+k_{h}\right)\right)}{\vartheta\left(\int_{1}^{p}\left(\int_{\gamma_{h}}^{z, s} d u_{h}-v_{h}+k_{h}\right)\right) \vartheta\left(\frac{p}{h}\left(\int_{1}^{z, s} d u_{h}-w_{h}+k_{h}\right)\right)},
$$

und zur Bestimmung der Verhältnisse der $a_{0}, a_{1}, \ldots, a_{p}$ ergeben sich die Gleichungen :

$$
\begin{gathered}
0=\sum_{i=0}^{i=p} a_{i} \vartheta_{i}\left(\begin{array}{l}
p \\
h
\end{array}\left(\int_{\gamma_{h}}^{c}{ }^{c} d u_{h}-\vartheta_{h}+k_{h}\right)\right) \vartheta_{i}\left(\underset{1}{h}\left(\int_{\gamma_{h}}^{c}{ }^{c} d u_{h}-w_{h}+k_{h}\right)\right), \\
\rho=1,2, \ldots p
\end{gathered}
$$

*) Diese Functionen $\vartheta_{1}, \vartheta_{2}, \ldots, \vartheta_{p}$ sind specielle Fälle von den von Roch und Prym benutzten, mit Charakteristiken verseheuen $\vartheta$-Functionen. Man könnte ebenso gut auch $p$ andere von diesen $\vartheta$-Functionen benutzen. (Roch, über die Doppeltangenten der Curven vierter Ordnung, Bd. 66 dieses Journals; Prym, Theorie der Funutionen in einer zweiblättrigen Fläche, Zürich 1866.) 
wo $\vartheta$ an die Stelle von $\vartheta_{0}$ zu setzen ist. Um wie im vorigen Paragraphen die ganze rationale Function $p^{\text {ten }}$ Grades $\psi(\sigma)(\$ .4$. (18.)) zu bilden, deren Wurzeln die $p$ Werthe $\sigma_{z_{1}}, \sigma_{z_{3}}, \ldots, \sigma_{z_{p}}$ der rationalen Function $\sigma$ von $s$ und $s$ in den Punkten $z_{1}, z_{2}, \ldots, z_{p}$ ergeben, hat man sich des dort angegebenen Verfahrens zu bedienen, indem man die in $\$$. 3 aufgestellte Formel für das Umkehrproblem zu Hülfe nimmt.

Nimmt die Function $\sigma$ die Werthe

$$
\begin{aligned}
& \sigma_{x_{1}}, \sigma_{x_{2}}, \ldots, \sigma_{x_{p}} \text { in den Punkten } x_{1}, x_{2}, \ldots, x_{p} \\
& \sigma_{y_{1}}, \sigma_{y_{2}}, \ldots, \sigma_{y_{p}} \quad \text { - } \quad \text { - } y_{1}, y_{2}, \ldots, y_{p} \\
& \sigma_{z_{1}}, \sigma_{z_{2}}, \ldots, \sigma_{z_{p}} \quad-\quad \text { - } z_{1}, z_{2}, \ldots, z_{p} \\
& \sigma_{c_{1}}, \sigma_{c_{2}}, \ldots, \sigma_{c_{p}}-\sigma_{x_{1}}-c_{1}, c_{2}, \ldots, c_{p}
\end{aligned}
$$

an, einen beliebigen Werth $\sigma$ in den Punkten $\xi_{1}, \xi_{2}, \ldots, \xi_{\mu}$ und den Werth $\propto$ in den Punkten $\xi_{1}^{(1)}, \xi_{2}^{(1)}, \ldots, \xi_{p}^{(1)}$, setzt man ferner zur Abkürzung:

$$
\begin{aligned}
& \psi^{(x)}(\sigma)=\left(\sigma-\sigma_{x_{1}}\right)\left(\sigma-\sigma_{x_{2}}\right) \ldots\left(\sigma-\sigma_{x_{p}}\right), \\
& \psi^{(y)}(\sigma)=\left(\sigma-\sigma_{y_{1}}\right)\left(\sigma-\sigma_{y_{3}}\right) \ldots\left(\sigma-\sigma_{y_{p}}\right), \\
& \psi^{(z)}(\sigma)=\left(\sigma-\sigma_{z_{1}}\right)\left(\sigma-\sigma_{z_{2}}\right) \ldots\left(\sigma-\sigma_{z_{p}}\right), \\
& \psi^{(c)}(\sigma)=\left(\sigma-\sigma_{c_{1}}\right)\left(\sigma-\sigma_{c_{3}}\right) \ldots\left(\sigma-\sigma_{c_{p}}\right),
\end{aligned}
$$

hat endlich die Function $\zeta$ in den Punkten $\xi_{1}, \xi_{2}, \ldots, \xi_{\mu}$ die Werthe $\zeta^{(1)}, \zeta^{(2)}, \ldots, \zeta^{(\mu)}$ und in den Punkten $\xi_{1}^{(1)}, \xi_{2}^{(1)}, \ldots, \xi_{\mu}^{(1)}$ die Werthe $\zeta_{10}^{(1)}, \zeta_{11}^{(2)}, \ldots, \zeta_{11}^{(\mu)}$, so ergiebt sich nach dem im vorigen Paragraphen auseinandergesetzten Verfahren die Formel

$$
\frac{\psi^{(z)}(\sigma)}{\psi^{(c)}(\sigma)}=\frac{\zeta^{(1)} \zeta^{(2)} \ldots \zeta^{(\mu)}}{\zeta_{0}^{(1)} \zeta_{0}^{(2)} \ldots \zeta_{0}^{(\mu)}} \frac{\psi^{(x)}(\sigma)}{\psi^{(c)}(\sigma)} \cdot \frac{\psi^{(v)}(\sigma)}{\psi^{(c)}(\sigma)} .
$$

Die beiden Quotienten $\frac{\psi^{(x)}(\sigma)}{\psi^{(c)}(\sigma)} ; \frac{\psi^{(y)}(\pi)}{\psi^{(c)}(\sigma)}$ sind aber durch die Formel (14.) $\$ .3$ durch 9-Functionen ausgedrückt, und zwar genau mit denselben Argumenten, welche nach den Resultaten dieses Paragraphen in den $\vartheta$-Functionen vorkommen, durch welche die Function $\zeta$ darstellbar ist.

Um aus der Formel (26.) die Coefficienten $M_{1}, M_{2}, \ldots, M_{p}$ der Function

$$
\psi^{(z)}(\sigma)=\sigma^{p}+M_{1} \sigma^{p-1}+\cdots+M_{p-1} \sigma+M_{p}
$$

zu finden, hat man nur nöthig, diese Gleichung aufzustellen für $p$ verschiedene Werthe von $\sigma$, denen dann $p$ Systeme von Punkten $\xi_{1}, \xi_{2}, \ldots, \xi_{\mu}$ entsprechen. Diese Coefficienten erhält man so rational ausgedrückt durch $\boldsymbol{\vartheta}$-Functionen, welche resp. nur von den Argumenten $v_{i}$ oder $w_{i}$ abhängig sind, diese Argumente vermehrt um gewisse Constanten. 


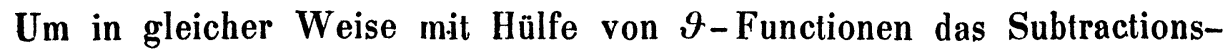
problem zu lösen, hat man nur durchweg $w_{1}, w_{2}, \ldots, w_{p}$ zu vertauschen mit $-w_{1},-w_{2}, \ldots,-w_{p}$. Es ist dabei aber $\mathrm{zu}$ beachten, dass die auf diese Weise aus $\zeta$ hervorgehende Function ' $\zeta$ " nicht identisch ist mit der im vorigen Paragraphen eingeführten Funclion ' $\zeta$ ', wiewohl ihr Verschwinden dieselben Punkte $z_{1}^{\prime}, z_{2}^{\prime}, \ldots, z_{p}^{\prime}$ ergiebt. Es wird nämlich die Funclion

$$
\begin{aligned}
& \zeta^{\prime}=0 \text { für } y_{1} \quad y_{2} \ldots y_{p} \quad z_{1}^{\prime} z_{2}^{\prime} \ldots \ldots z_{p}^{\prime} \\
& \zeta=\infty \text { für } \begin{array}{ccccccccc}
c_{1} & c_{2} & \ldots & c_{p} & x_{1} & x_{2} & \ldots & x_{p},
\end{array} \\
& \zeta^{\prime \prime}=\begin{array}{lllllllllll}
0 & \text { für } & c_{1} & c_{2} & \ldots & c_{p} & z_{1}^{\prime} & z_{2}^{\prime} & \ldots & z_{p}^{\prime}
\end{array} \\
& \zeta=\infty \text { für } y_{1}^{\prime} y_{2}^{\prime} \ldots y_{p}^{\prime} \quad x_{1} x_{2} \ldots x_{p} \text {, }
\end{aligned}
$$

wo die $y_{1}^{\prime}, y_{2}^{\prime}, \ldots, y_{p}^{\prime}$ neue Punkte sind, welche mit den Punkten $y_{1}, y_{2}, \ldots, y_{p}$ verbunden sind durch die Bedingungen:

$$
\begin{aligned}
& \int_{c_{1}}^{y_{1}} d u_{Q}+\int_{c_{2}}^{y_{2}} d u_{Q}+\cdots+\int_{c_{p}}^{y_{p}} d u_{\varphi}+\int_{c_{1}}^{y_{1}^{\prime}} d u_{Q}+\int_{c_{Q}}^{y_{2}^{\prime}} d u_{Q}+\cdots+\int_{c_{p}}^{y_{p}^{\prime}} d u_{Q}=0 . \\
& \varrho=1,2 \ldots p
\end{aligned}
$$

Man ersieht hieraus, dass die Punkte $y_{1}^{\prime}, y_{2}^{\prime}, \ldots, y_{p}^{\prime}$ mittelst der Function $\zeta^{\prime}$

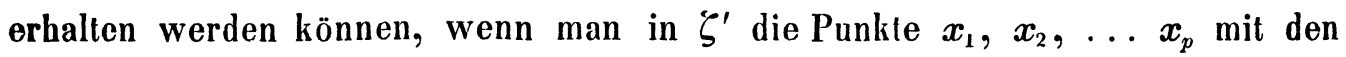
$c_{1}, c_{2}, \ldots, c_{p}$ zusammenfallen lässt. Die daraus hervorgehende Function

$$
\zeta_{0}^{\prime} \quad \text { wird }=0 \begin{array}{lllllllllll}
\text { für } & y_{1} & y_{2} & \ldots & y_{p} & y_{1}^{\prime} & y_{2}^{\prime} & \ldots & y_{p}^{\prime} \\
- & =\infty & - & c_{1} & c_{2} & \ldots & c_{p} & c_{1} & c_{2} & \ldots & c_{p}
\end{array}
$$

und daraus folgt, dass die hier durch $\vartheta$-Functionen gebildete Function $\zeta^{\prime \prime}$ den algebraischen Ansdruck haben muss:

$$
\text { (28.) } \quad \zeta^{\prime \prime}=\frac{\zeta^{\prime}}{\zeta_{0}^{\prime}},
$$

abgesehen von einem constanten Factor. Die Function ' $\zeta_{1}^{\prime}$, welche dazu dient, aus dem als gelōst vorausgesetzten Unkehrproblem für gewisse Werthe der Argumente dasselbe zu lösen für die entgegengesetzten Werthe, gestatten einen sehr einfachen Ausdruck durch $\vartheta$-Functionen:

$$
\zeta_{0}^{\prime}=\frac{\vartheta\left(\begin{array}{l}
p \\
h
\end{array}\left(\int_{\gamma_{h}}^{z, s} d u_{h}-w_{h}+k_{h}\right)\right) \vartheta\left(\begin{array}{l}
p \\
h
\end{array}\left(\int_{\gamma_{h}}^{z, s} d u_{h}+w_{h}+k_{h}\right)\right)}{\left(\vartheta\left(\begin{array}{l}
p \\
h
\end{array}\left(\int_{\gamma_{h}}^{z, s} d u_{h}+k_{h}\right)\right)\right)^{2}},
$$

so dass durch die Formel (28.) auch ' $\zeta$ ' ausgedrückt ist durch $\boldsymbol{\vartheta}$-Functionen, die nur von den Argumenten $v_{i}$ oder nur von den Argumenten $w_{i}$ abhängen. 
S. 6.

Das Additionstheorem für die zweite und dritte Gattung.

Mit Hülfe derselben Function $\zeta$, die in den beiden letzten Paragraphen auf algebraischem und auf transcendentem Wege bestimmt wurde, lässt sich eine Reihe von Additionsformeln für gewisse Summen von Integralen zweiter und dritter Gattung aufstellen, die vollständig analog sind den Formeln, welche Jacobi für die Addition der elliptischen Transcendenten zweiter und dritter Gattung angegeben hat.

Es werde wie oben (\$. 3) gesetzt:

$$
P_{\left(\varepsilon_{1} \varepsilon_{2}\right)}\left(v_{1}, v_{2}, \ldots, v_{p}\right)=\int_{c_{1}}^{x_{1}} d \pi_{\left(\varepsilon_{1} \varepsilon_{2}\right)}+\int_{c_{2}}^{x_{2}} d \pi_{\left(\varepsilon_{1} \varepsilon_{2}\right)}+\cdots+\int_{c_{p}}^{x_{p}} d \pi_{\left(\varepsilon_{1} \varepsilon_{2}\right)}
$$

und ebenso als Transcendente zweiter Gattung:

(30.) $\quad Z_{(\varepsilon)}\left(v_{1}, v_{2}, \ldots, v_{p}\right)=\int_{c_{1}}^{x_{1}} d t_{(\varepsilon)}+\int_{c_{2}}^{x_{2}} d t_{(\varepsilon)}+\cdots+\int_{c_{p}}^{x_{p}} d t_{(\varepsilon)}$.

Es sind hierin $P$ und $Z$ anzusehen als Functionen von

$$
\begin{gathered}
\text { (31.) } \quad v_{\varrho}=\int_{c_{1}}^{x_{1}} d u_{\varphi}+\int_{c_{2}}^{x_{2}} d u_{\rho}+\cdots+\int_{c_{p}}^{x_{p}} d u_{\rho} . \\
\varrho=1,2 \ldots p
\end{gathered}
$$

Werden die Integrationswege in (29.), (30.), (31.) gleich angenommen, so ist $Z$ eine völlig eindeutige Function der $v_{1}, v_{2}, \ldots, v_{p}$, welche von der Unstetigkeitsstelle $\varepsilon$ algebraisch abhängt, während $P$ für ein bestimmtes Werthsystem von $v_{1}, v_{2}, \ldots, v_{p}$ nur bis auf ganze Vielfache von $2 \pi i$ bestimmt ist.

Der Ausdruck für $\boldsymbol{P}$ durch $\mathscr{\vartheta}$-Functionen wurde oben schon aufgestellt:

(32.)

$$
P_{\left(\varepsilon_{1} \varepsilon_{2}\right)}\left(v_{1}, v_{2}, \ldots, v_{p}\right)=\log \frac{\vartheta\left(\begin{array}{l}
p \\
h
\end{array}\left(\int_{\gamma_{h}}^{\varepsilon_{2}} d u_{h}-v_{h}+k_{h}\right)\right) \vartheta\left(\begin{array}{l}
p \\
h
\end{array}\left(\int_{1}^{\varepsilon_{1}} d u_{h}+k_{h}\right)\right)}{\vartheta\left({ }_{1}^{p}\left(\int_{\gamma_{h}}^{\varepsilon_{1}} d u_{h}-v_{h}+k_{h}\right)\right) \vartheta\left({ }_{1}^{p}\left(\int_{\gamma_{h}}^{\varepsilon_{2}} d u_{h}+k_{h}\right)\right)},
$$

und ebenso ergiebt sich aus den Formeln von Riemann (1. c. \$. 25)

(33.) $Z_{(\varepsilon)}\left(v_{1}, v_{2}, \ldots, v_{p}\right)=$

$$
\partial\left[\log \vartheta\left(\underset{1}{h}\left(\int_{\gamma_{h}}^{\varepsilon} d u_{h}-v_{h}+k_{h}\right)\right)-\log \vartheta\left(\underset{1}{h}\left(\int_{\gamma_{h}}^{\varepsilon} d u_{h}+k_{h}\right)\right)\right]
$$

Man erhält $\boldsymbol{P}_{\left(\varepsilon_{1} \varepsilon_{2}\right)}$ aus $\boldsymbol{Z}_{(\varepsilon)}$ durch Integration in Bezug auf den Unstetigkeitswerth zwischen den Grenzen $\varepsilon_{1} \varepsilon_{2}$ und umgekehrt $Z_{(\varepsilon)}$ aus $P_{\left(\varepsilon_{1} \varepsilon_{2}\right)}$ durch Differentiation nach dem Unstetigkeitspunkt $\varepsilon_{1}$. 
Setzt man nun der Definition gemäss

$$
\begin{aligned}
& Z_{(\varepsilon)}\left(v_{1}, v_{2}, \ldots, v_{p}\right)=\sum_{i=1}^{i=p} \int_{c_{i}}^{x_{i}} d t_{(\varepsilon)}, \\
& Z_{(\varepsilon)}\left(w_{1}, w_{2}, \ldots, w_{p}\right)=\sum_{i=1}^{i=p} \int_{c_{i}}^{y_{i}} d t_{(\varepsilon)}, \\
& Z_{(\varepsilon)}\left(v_{1}+w_{1}, v_{2}+w_{2}, \ldots, v_{p}+w_{p}\right)=\sum_{i=1}^{i=p} \int_{c_{i}}^{z_{i}} d t_{c},
\end{aligned}
$$

so ergiebt sich daraus:

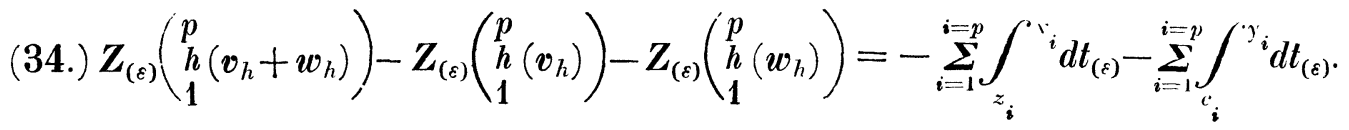

Berücksichtigt man den Zusammenhang, welcher zwischen den Punkten $x_{i} y_{i}$ $z_{i} c_{i}$ besteht, dass nämlich die. Function $\zeta$ in $x_{i} y_{i}$ unendlich, in $z_{i} c_{i}$ Null wird, so lässt sich auf die rechte Seite der Gleichung (34.) das Abelsche Theorem anwenden ( $\$ .1(2)$.$) , und man gelangt so zu der Additionsformel für$ die Function $Z$ :

$$
\text { (35.) } \quad \boldsymbol{Z}_{(\varepsilon)}\left(\begin{array}{l}
p \\
h \\
1
\end{array}\left(\boldsymbol{v}_{h}+\boldsymbol{w}_{h}\right)\right)-\boldsymbol{Z}_{(\varepsilon)}\left(\begin{array}{l}
p \\
\boldsymbol{1}
\end{array}\left(\boldsymbol{v}_{h}\right)\right)-\boldsymbol{Z}_{(\varepsilon)}\left(\begin{array}{l}
p \\
\boldsymbol{h}
\end{array}\left(\boldsymbol{w}_{h}\right)\right)=-\frac{\partial \log \zeta_{(\varepsilon)}}{\partial \boldsymbol{z}_{(\varepsilon)}} .
$$

Man erhält ferner, entweder auf demselben Wege, oder auch aus der Formel (35.) durch Integration die Additionsformel für die Function $\boldsymbol{P}_{\left(\varepsilon_{1} \varepsilon_{2}\right)}$ :

(36.) $\quad \boldsymbol{P}_{\left(\varepsilon_{1} \varepsilon_{2}\right)}\left(\begin{array}{l}p \\ \left.h\left(v_{h}+w_{h}\right)\right)-P_{\left(\varepsilon_{1} \varepsilon_{2}\right)} \\ 1\end{array}\left(\begin{array}{l}p \\ h \\ 1\end{array}\left(v_{h}\right)\right)-P_{\left(\varepsilon_{1} \varepsilon_{2}\right)}\left(\begin{array}{l}p \\ h \\ 1\end{array}\left(w_{h}\right)\right)=\log \zeta_{\varepsilon_{3}}-\log \zeta_{\varepsilon_{1}}\right.$.

Durch eine ganz entsprechende Betrachtung erhält man für die Subtraction die beiden folgenden Formeln, in denen ' $\zeta$ ' dieselbe Function wie oben bedeutet:

(37.) $\boldsymbol{Z}_{(\varepsilon)}\left(\begin{array}{l}p \\ h\end{array}\left(v_{h}-w_{h}\right)\right)-Z_{(\varepsilon)}\left(\underset{1}{h}\left(v_{h}\right)\right)+Z_{(\varepsilon)}\left(\underset{1}{h}\left(w_{h}\right)\right)=-\frac{\partial \log \zeta_{(\varepsilon)}^{\prime}}{\partial z_{(\varepsilon)}}$,

(38.) $\quad P_{\left(\varepsilon_{1} \varepsilon_{2}\right)}\left(\underset{1}{h}\left(v_{h}-w_{h}\right)\right)-P_{\left(\varepsilon_{1} \varepsilon_{2}\right)}\left(\begin{array}{l}p \\ h \\ 1\end{array}\left(v_{h}\right)\right)+P_{\left(\varepsilon_{1} \varepsilon_{2}\right)}\left(\underset{1}{h}\left(w_{h}\right)\right)=\log \zeta_{\left(\varepsilon_{2}\right)}^{\prime}-\log \zeta_{\left(\varepsilon_{1}\right)}^{\prime}$.

Setzt man in den beiden letzteren Formeln $v_{1}=v_{2}=\cdots=v_{p}=0$, lässt man mit andern Worten die Punkte $x_{i}$ in die $c_{i}$ hineinfallen, so ergiebt sich:

(39.) $\quad Z_{(\varepsilon)}\left(-w_{1},-w_{2}, \ldots,-w_{p}\right)+Z_{(\varepsilon)}\left(w_{1}, w_{2}, \ldots, w_{p}\right)=-\frac{\partial \log \zeta_{(\varepsilon)}^{\prime}}{\partial z_{\varepsilon}}$, (40.) $P_{\left(\varepsilon_{1} \varepsilon_{2}\right)}\left(-w_{1},-w_{2}, \ldots,-w_{p}\right)+P_{\left(\varepsilon_{1} \varepsilon_{2}\right)}\left(w_{1}, w_{2}, \ldots, w_{p}\right)=\log \zeta_{0\left(\varepsilon_{2}\right)}^{\prime}-\log \zeta_{0\left(\varepsilon_{1}\right)}^{\prime}$, 
wo $\zeta_{0}^{\prime}$ dieselbe Function ist, die im vorigen Paragraphen eingeführt und dort durch $9-$ Functionen ausgedrückt wurde.

Die Verbindung der Formeln (35.), (36.), (37.), (38.) führt endlich noch zu folgenden Relationen:
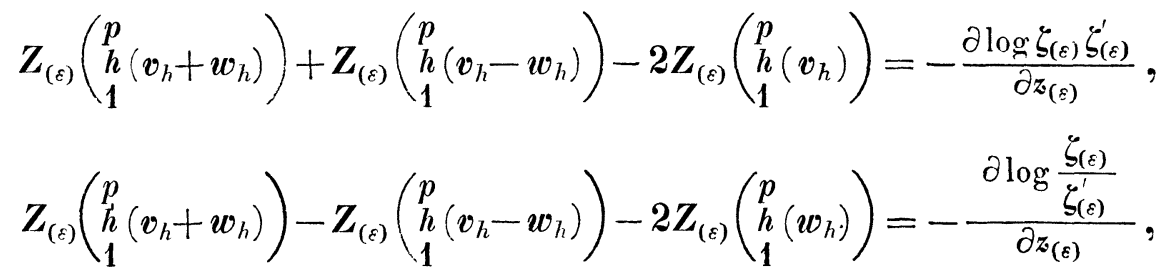

$$
\begin{gathered}
\left\{\begin{array}{c}
P_{\left(\varepsilon_{1} \varepsilon_{2}\right)}\left(\begin{array}{l}
p \\
\left.h\left(v_{h}+w_{h}\right)\right) \\
1
\end{array}\right) P_{\left(\varepsilon_{1} \varepsilon_{2}\right)}\left(\begin{array}{l}
p \\
h \\
1
\end{array}\left(v_{h}-w_{h}\right)\right)-2 P_{\left(\varepsilon_{1} \varepsilon_{2}\right)}\left(\begin{array}{l}
p \\
h \\
1
\end{array}\left(v_{h}\right)\right) \\
=\log \zeta_{\left(\varepsilon_{2}\right)} \zeta_{\left(\varepsilon_{2}\right)}^{\prime}-\log \zeta_{\left(\varepsilon_{1}\right)} \zeta_{\left(\varepsilon_{1}\right)}^{\prime},
\end{array}\right. \\
\left\{\begin{array}{c}
P_{\left(\varepsilon_{1} \varepsilon_{2}\right)}\left(\begin{array}{l}
p \\
h\left(v_{h}+w_{h}\right) \\
1
\end{array}\right)-P_{\left(\varepsilon_{1} \varepsilon_{2}\right)}\left(\begin{array}{l}
p \\
h\left(v_{h}-w_{h}\right)
\end{array}\right)-2 P_{\left(\varepsilon_{1} \varepsilon_{2}\right)}\left(\begin{array}{l}
p \\
h \\
1
\end{array}\left(w_{h}\right)\right) \\
=\log \frac{\zeta_{\left(\varepsilon_{2}\right)}}{\zeta_{\left(\varepsilon_{2}\right)}^{\prime}}-\log \frac{\zeta_{\left(\varepsilon_{1}\right)}}{\zeta_{\left(\varepsilon_{1}\right)}^{\prime}} .
\end{array}\right.
\end{gathered}
$$

Alle diese Additionsformeln, die sich auf die zweite und dritte Gattung beziehen, sind unabhängig von der Voraussetzung, dass die zu Grunde gelegten Integrale zweiter und dritter Gattung gerade die oben als Normalintegrale bezeichneten seien; nur die obigen Ausdrücke durch die 9 -Functionen sind auf diese Voraussetzung beschränkt. Man erkennt in diesen Formeln leicht die Analogien zu den von Jacobi in den "Fundamenta nova theoriae functionum ellipticarum" aufgestellten Additionsformeln für die elliptischen Integrale zweiter und dritter Gattung.

Heidelberg, im März 1869. 